\title{
Hibrid 3B-2B ESA Mimarisi Kullanılarak Hiperspektral Uzaktan Algılama Görüntülerinin Sınıflandırılması
}

\author{
Classification of Hyperspectral Remote Sensing Images Using Hybrid \\ 3D-2D CNN Architecture \\ Hüseyin FIRAT $^{* 1}$ iD , Murat UÇAN ${ }^{1}$ (D) , Davut HANBAY2 ${ }^{2}$ \\ ${ }^{1}$ Bilgisayar Teknolojileri Bölümü, Dicle Üniversitesi-Tenik Bilimler MYO, Diyarbakır, Türkiye \\ 2Bilgisayar Mühendisliği Bölümü, İnönü Üniversitesi, Malatya, Türkiye \\ (hossein.firat@gmail.com, murat.ucan@dicle.edu.tr, davut.hanbay@inonu.edu.tr)
}

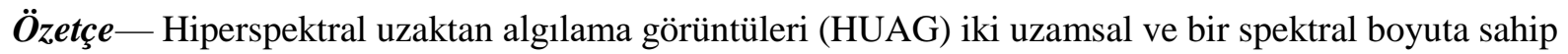
görüntü küpleridir. Evrişimsel sinir ağı (ESA), HUAG sınıflandırmada uzamsal-spektral özellik bilgilerinin çıkarılmasını sağlayan en etkili derin öğrenme yöntemlerinden biridir. Geleneksel ESA tabanlı yöntemler genellikle özellik çıkarımı için 2B ESA'yı kullanmaktadır. 2B ESA yalnızca uzamsal boyutttaki özellikleri yakaladığından dolayı tek başına spektral boyutlardan iyi ayırt edici özellik haritaları çıkaramaz. 3B ESA HUAG'deki uzamsal-spektral özellikleri eş zamanlı olarak çıkarabilmektedir. Ancak 3B ESA hesaplama açısından karmaşıktır. Bu çalışmada 3B-2B ESA birleşiminden oluşan hibrid bir yöntem önerilmiştir. 3B ESA ortak uzamsal-spektral özellikleri çıkarırken, 3B ESA'dan sonra kullanılan 2B ESA ile daha fazla uzamsal özellikler öğrenilir. Ayrıca önerilen hibrid yöntem hesaplama karmaşıklığını da azaltmaktadır. Önerilen yöntemin sınıflandırma performansını arttırmak için Mish aktivasyon fonksiyonu kullanılmaktadır. Yaygın olarak kullanılan iki veriseti ile gerçekleştirilen uygulamalar sonucunda önerilen yöntemin daha iyi sınıflandırma performansına sahip olduğu görülmektedir.

Anahtar Kelimeler : Uzaktan Algılama, Hiperspektral Görüntü Sinıflandırma, Derin ögrrenme.

Abstract - Hyperspectral remote sensing images (HRSI) are image cubes with two spatial and one spectral dimensions. Convolutional neural network (CNN) is one of the most effective deep learning methods for extracting spatial-spectral feature information in HRSI classification. Traditional CNNbased methods usually use 2D CNN for feature extraction. Because 2D CNN captures only spatialdimensional features, it cannot extract good feature maps from spectral dimensions. 3D CNN can simultaneously extract spatial-spectral features in HRSI. However, 3D CNN is computationally complex. In this study, a hybrid method consisting of 3D-2D CNN combination is proposed. While 3D CNN extracts common spatial-spectral features, more spatial features are learned with 2D CNN used after 3D CNN. In addition, the proposed hybrid method reduces the computational complexity. Mish activation function is used to increase the classification performance of the proposed method. As a result of the applications performed with two commonly used datasets, it is seen that the proposed method has better classification performance.

Keywords : Remote Sensing, Hyperspectral Image Classification, Deep Learning. 


\section{Giriş}

Hiperspektral uzaktan algılama görüntüleri (HUAG) genellikle görünürden kızılötesi spektruma kadar uzanan yüzlerce spektral bant hakkında bilgi içermektedir (Meng et al., 2019). Yüzlerce spektral bantta bulunan zengin spektral bilgilerle, HUAG, arazi örtüsü analizi, askeri gözetim, nesne tespiti, tarım ve madencilik vb. çok çeşitli uzaktan algılama uygulamalarında başarıyla uygulanmaktadır (Jia et al., 2020; Firat and Hanbay, 2021). Bu uygulamalar arasında, görüntü sinıflandırma, HUAG'daki her pikseli benzersiz bir anlamsal kategori veya sınıfa atamayı amaçlamaktadır. Bununla birlikte, HUAG'nin yüksek boyutluluğu, sınıflandırma için önemli bir probleme neden olmaktadır. HUAG sınıflandırma yöntemlerinin çoğu, yüksek boyutluluk için bir çözüm olarak boyut indirgeme yöntemlerini kullanmaktadır. Temel bileşen analizi (TBA) (Kang et al., 2020), doğrusal diskriminant analizi (Xu et al., 2019) ve bağımsız bileșen analizi (Villa et al., 2011) gibi boyut indirgeme yöntemleri ile HUAG'deki yararlı özellikleri çıkarmak amaçlanmaktadır. Bu işlem sonucunda veri boyutu düşürülmekte ve işlem maliyeti azaltılmaktadır. TBA, HUAG sınıflandırması için en yaygın kullanılan boyut indirgeme yöntemidir. TBA, doğrusal bir yöntemdir ve diğer boyut indirgeme yöntemlerine göre hesaplama açısından daha az karmaşıktır.

Son yıllarda kendi kendine öğrenen sınıflandırıcıların yardımıyla veya manuel olarak gerçekleştirilen çok sayıda HUAG sınıflandırma yöntemi önerilmiştir. Geleneksel HUAG sınıflandırma yöntemleri genellikle yalnızca spektral bilgilere dayanmaktadır. Bu sınıflandırıcılar arasında lojistik regresyon (Ahmad et al., 2020), destek vektör makinesi (DVM) (Wang et al., 2020) ve rastgele orman (Ham et al., 2005) temelli sinıflandırıcılar bulunmaktadır. Bu sinıflandırıcılar, spektral fazlalık ve spektral bantlar arasındaki yüksek korelasyon nedeniyle iyi performans gösterememektedir. Ayrıca, bu sınıflandırıcılar HUAG'nin önemli uzamsal değişkenliğini korumada başarısız olmaktadır. Bu da düşük performansla sonuçlanmaktadır. Geleneksel sınıflandırıcı yöntemlerinin çoğu, özellikleri manuel olarak çıkarmakta ve bir uzmanın yardımına ihtiyaç duymaktadır. Derin öğrenme tekniklerindeki ilerleme, HUAG sınıflandırmasını yeni bir aşamaya taşımaktadır. Derin öğrenme yönteminin eğitim aşaması, özellikleri otomatik olarak çıarmakta ve bunları sınıflandırma aşamasında kullanmaktadır (Mohan and Venkatesan, 2020). Tipik bir derin öğrenme modeli olarak, yığınlı otokodlayıcı (Zhang et al., 2016) ve derin inanç ağı (Chen et al., 2015) hem uzamsal hem de spektral bilgiyi çıkarabilmekte ve daha sonra bunları HUAG sınıflandırması için birleştirebilmektedir. Bununla birlikte, yukarıdaki derin öğrenme yöntemlerinin ikisi de, girdi olarak tek boyutlu özellik vektörlerini kullanmaktadır. Bu durumda HUAG' deki uzamsal özellikler tam olarak kullanılamamaktadır. HUAG sınıflandırmada, hem uzamsal hem de spektral alanlardan gizli özelliklerin çıkarılmasında kullanılan en etkili derin öğrenme yöntemlerden biri de evrişimsel sinir ağlarıdır (ESA) (Üzen et al., 2021). ESA, daha yüksek kalitede HUAG sınıflandırması için daha ayırt edici özellikler sağlayabilen güçlü bir özellik öğrenmeye sahiptir. (Mingyi He, Bo Li, 2017) nolu çalışmada, 2 boyutlu çok ölçekli uzamsal özellikleri ve 1 boyutlu spektral özellikleri birlikte öğrenen çok ölçekli 3 boyutlu derin evrişimsel sinir ağı (M3B-ESA) önerilmiştir. (Roy et al., 2019) nolu çalışmada, HUAG'lerin uzamsal ve spektral özelliklerini etkin bir şekilde çıkaran 2B ESA ile 3B ESA birleştirilerek hibrit bir ağ modeli (HybridSN) tasarlanmıştır. (Zhong et al., 2018) nolu çalışmada, denetimli bir model olan HUAG sınıflandırması için spektral-uzamsal artık ağ (SSRN) önerilmiştir. (Y. Chen, H. Jiang, C. Li, X. Jia, 2016) nolu çalışmada, HUAG sinıflandırması için 3B ESA kullanılmıştır. Hiperspektral görüntülerin hacimsel veri olması ve spektral boyuta sahip olmasından dolayı 2B ESA spektral boyutlardan iyi ayırt edici özellik haritaları çıkaramamaktadır. Çünkü 2B ESA ile uzamsal özellikler genellikle ayrı olarak çıkarılmakta ve uzamsal-spektral bilgiden ortak olarak yararlanmayı bir dereceye kadar geçersiz kılmaktadır. Artan hesaplama karmaşıklığı nedeniyle çok az yöntem 3B ESA kullanmaktadır. 3B ESA ile spektral ve uzamsal özellikler eşzamanlı olarak çıkarıldığından, 3B HUAG'lerin yapısal özelliklerinden tam olarak yararlanılmaktadır. Bu çalışma kapsamında, mümkün olabilecek maksimum doğruluğu elde etmek için hem spektral hem de uzamsal özellik haritalarını tam olarak kullanacak şekilde 3B ESA ile 2B ESA'nın birleşiminden oluşan hibrid bir ESA yöntemi önerilmektedir. HUAG sınıflandırması için önerilen yöntemin önemli katkıları aşağıdaki gibidir:

1. 3B ESA hem uzamsal hem de spektral özellikleri çıkarsa da, çok sayıda eğitim parametresi nedeniyle hesaplama açısından karmaşıktır. 3B ESA'nın üzerinde 2B ESA kullanımı, hesaplama karmaşıklığını azaltır ve daha fazla uzamsal özellik öğrenir. 
2. Relu aktivasyon fonksiyonunun yerine yeni bir aktivasyon fonksiyonu olan Mish kullanılır.

3. HUAG'lerinin yüksek boyutluluğundan oluşan hesaplama karmaşıklığını azaltmak için boyut indirgeme yöntemi olarak TBA kullanılır.

4. Önerilen Hibrit 3B-2B ESA yöntemi, daha fazla uzamsal özellik çıkarır ve mevcut derin öğrenme yöntemlerine kıyasla sınıflandırma doğruluğunu arttırır.

Bu çalışmanın geri kalanının düzeni şu şekildedir. Bölüm 2'de önerilen yöntem, Bölüm 3'te uygulamalarda kullanılan verisetleri ve elde edilen uygulama sonuçları yer almaktadır. Bölüm 4'te ise makalenin genel bir özeti verilmektedir.

\section{2. Önerilen Yöntem}

Hiperspektral görüntü $X, M \times N \times D$ boyutunda 3 boyutlu bir küp olarak belirtilmiştir. Burada $M$ ve $N$, görüntünün uzamsal genişliği ve yüksekliğidir. $D$ ise, spektral bantların sayısını belirtir. Giriş görüntüsünün ground truth'u $Y$, one-hot encoding kullanılarak dönüştürülür ve $Y=\left(y_{1}, y_{2}, \ldots \ldots, y_{C}\right)$ olarak gösterilir. Burada $C$, girdi görüntüsünde bulunan sınıfların sayısını belirtmektedir. HUAG pikselleri, herhangi bir sınıflandırma modeli için yoğun çaba gerektiren yüksek sınıflar arası benzerlik, yüksek sınıf içi değişkenlik, üst üste binen ve iç içe bölgeler sergilemektedir. Bu sorunların üstesinden gelmek için spektral fazlalığı gidermek gerekmektedir. Spektral fazlalığını gidermek için ilk önce orjinal HUAG verilerine spektral bantlar boyunca geleneksel TBA uygulanır. TBA, aynı uzamsal boyutları (yani, genişlik M ve yükseklik N) korurken spektral bant sayısını D'den B'ye düşürür. Herhangi bir nesneyi tanımak için çok önemli olan uzamsal bilgiyi koruyacak şekilde yalnızca spektral bantları azaltılmış oldu. Böylelikle, istenilen bant sayısına indirgenmiş oldu. Görüntü sınıflandırma tekniklerini kullanmak için, hiperspektral veri küpü, merkezi piksele dayalı gerçek etiketlerin oluşturulduğu küçük üst üste binen 3B uzamsal parçalara bölünmüştür. Uzamsal konumda $(\mathrm{a}, \mathrm{b})$ merkezlenmiş ve $S x S$ penceresini veya uzamsal boyutu ve tüm $B$ spektral bantlarını kapsayan hiperspektral veri küpünden 3B komşu parçaları $S X S X B$ oluşturuldu. Hiperspektral veri küpünden üretilen toplam 3B parça sayısı (n), $(M-S+1) x(N-S+1)$ ile bulunur. 2B ESA yönteminde, giriş verilerini aktivasyon fonksiyonundan geçirmeden önce 2B çekirdek kullanarak evrişim işlemi gerçekleştirilmektedir. Evrişim, giriş verileri ile çekirdek arasındaki iç çarpımın toplamını hesaplayarak gerçekleşir. Çekirdek, tüm uzamsal boyutu kapsayacak şekilde girdi verilerinin üzerinde ilerlemektedir. Bu evrişim işlemi, giriş görüntüsünden uzamsal özellikleri çıkarmaya yardımcı olmaktadır. Her nöronun 2B evrişim çıktısı Denklem (1)'deki gibi formüle edilmektedir (Mohan and Venkatesan, 2020).

$$
y_{m n}=f\left(\sum_{r} \sum_{i=0}^{h-1} \sum_{j=0}^{w-1} k_{i j} x_{(i+m)(j+n)}+\text { bias }_{m n}\right)
$$

Denklem (1)'de $y_{m n},(m, n)$ konumunda çıkarılan özelliktir. $k, h \times w$ boyutundaki 2B evrişim çekirdeğidir. 2B görüntü olması durumunda, bu evrişim işlemi, alıcı alandaki tüm özellik haritaları $(r)$ üzerinde gerçekleştirilmekte ve doğrusal olmayan aktivasyon için tüm değerlerin toplamını almaktadır. $\mathrm{Bu}$ işlem, çok boyutlu veriler durumunda tüm katmanlar için tekrarlanmaktadır. Veriler üç boyutlu olduğunda bunların hem uzamsal hem de spektral veya zamansal boyutları vardır. Bu giriş verileri için 2B evrişim başarısız olmaktadır. Çünkü, geleneksel 2B ESA'da, evrişim işlemleri yalnızca uzamsal boyuttaki özellikleri yakalayan 2B özellik haritalarına uygulanmaktadır. 3B ESA, 2B ESA'da 2B evrişim yerine $3 \mathrm{~B}$ evrişim gerçekleştiren 2B ESA modellerinin değiştirilmiş şeklidir. Evrişim işlemleri, 3B verilere uygulandığında hem uzamsal hem de spektral boyutlardan özelliklerin yakalanması istenilmektedir. Bu amaçla, 3B giriş verilerinden ortak uzamsal spektral özellikleri hesaplamak için 3B evrişim işlemlerinin 3B özellik küplerine uygulandığ 1 3B ESA kullanılmaktadır. 3B evrişim, 3B spektral görüntülerden uzamsal-spektral özelliklerin çıkarılmasına yardımcı olmaktadır. 3B ESA yönteminden çıkarılan özellik Denklem (2)'deki gibi formüle edilmektedir (Mohan and Venkatesan, 2020).

$$
y_{m n p}=f\left(\sum_{r} \sum_{i=0}^{h-1} \sum_{j=0}^{w-1} \sum_{l=0}^{b-1} k_{i j l} x_{(i+m)(j+n)(l+p)}+\text { bias }_{m n p}\right)
$$


Denklem (2)'de $b$ spektral boyut boyunca 3B çekirdeğin boyutudur. Çekirdek ( $k$ ), üç boyutludur ve özellikler, 3B giriş verileri üzerinde 3B evrişim gerçekleştirilerek hesaplanmaktadır. Önerilen ağ mimarisinde, performansı daha da iyileştirmek için yeni bir aktivasyon fonksiyonu Mish kullanılmıştır. ReLU, çoğu HUAG sınıflandırma görevinde ve hatta çoğu derin öğrenme alanında yaygın olarak kullanılan bir aktivasyon fonksiyonudur. Bununla birlikte, yapılan testte ReLU'ya göre \%1.671 doğruluk artışına sahip olan (Misra, 2019) nolu çalışmada yeni bir derin öğrenme aktivasyon fonksiyonu Mish önerilmiştir. Mish'in hesaplama denklemi Denklem (3) ve (4)’teki gibi formüle edilmektedir.

$$
\begin{gathered}
\text { Mish }=x * \tanh \left(\operatorname{In}\left(1+e^{x}\right)\right) \\
\tanh (x)=\frac{e^{x}-e^{-x}}{e^{x}+e^{-x}}
\end{gathered}
$$

Denklem (3)'te $x$ fonksiyonun girdisidir.In(.) logaritmik bir hesaplamadır ve $\tanh ($.$) 'in hesaplanmas1$ Denklem (4)'te gösterilmektedir. Mish fonksiyonunun görüntüsü Şekil 1'de gösterilmektedir.

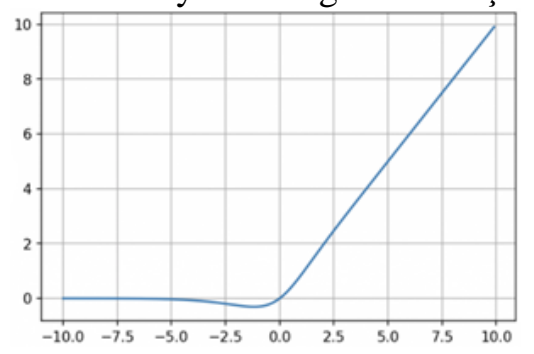

Şekil 1. Mish aktivasyon fonksiyonu (Ge et al., 2020)

Şekil 1'de gösterildiği gibi sınırsız olmak, Mish'in arzu edilen bir özelliğidir, çünkü doygunluğu önler ve teorik olarak, negatif değerler için hafif bir pay, ReLU'da katı bir sıfır sınırı yerine daha iyi gradyan akışına yol açar. Mish, daha iyi gradyan optimizasyonunu garanti eden sürekli bir aktivasyon fonksiyonudur. Bu özellikler, Mish kullanan yöntemlerin daha iyi sınıflandırma doğruluğuna sahip olmasinı sağlar (Ge et al., 2020).

Geleneksel 2B ESA'larda, 2B ayırt edici özellik haritalarını elde etmek için evrişim işlemi yalnızca uzamsal boyutlar üzerine uygulanır ve önceki katmanın tüm özellik haritalarını kapsar. Bununla birlikte HUAG sınıflandırma için, uzamsal özelliklerin yanında spektral özellik bilgilerinin de elde edilmesi istenilmektedir. 2B ESA'lar spektral özellik bilgilerini işleyemez. Diğer yandan, 3B ESA çekirdeği spektral ve uzamsal özellik bilgilerini, artan hesaplama karmaşıklığına rağmen HUAG verilerinden eşzamanlı olarak çıkarabilir. Hem 2B hem de 3B ESA'nın otomatik özellik öğrenme özelliğinin avantajlarından yararlanmak için, HUAG sınıflandırması kapsamında 3B ve 2B ESA'dan oluşan hibrid bir ESA yöntemi önerilmektedir. Önerilen 3B-2B ESA yönteminin akış diyagramı Şekil 2'de gösterilmektedir. Önerilen yöntem, 4 tane 3B ESA, 2 tane 2B ESA, düzleştirme, 2 tane Fully connected (FC), dropout ve softmax katmanlarından oluşmaktadır. Maksimum spektral ve uzamsal bilgiyi korumak için ESA'daki havuz katmanı kullanılmamaktır. Uygulanan 3B evrişim çekirdeklerinin boyutları ve kullanılan filtreler şu şekildedir. 1. evrişimli katmanda 7x7x7 (yani; iki uzamsal ve bir spektral boyut) boyutunda 8 filtre, 2. evrişim katmanında $5 \times 5 \times 5$ boyutunda 16 filtre, 3 . evrişim katmanında $3 \times 3 \times 3$ boyutunda 32 filtre ve 4.evrişim katmanında $1 \times 1 \times 1$ boyutunda 64 filtre kullanılmaktadır. 2B evrişim çekirdeklerinin boyutları ve kullanılan filtreler ise şu şekildedir. 1. evrişim katmanında $3 \times 3$ boyutunda 32 filtre ve 2 .evrişim katmanında $1 \times 1$ boyutunda 64 filtre kullanılmaktadır. Tüm evrişimli katmanlarda padding = 'valid' kullanılır, böylece her evrişimden sonra özellik haritasının boyutu azaltılmış olur. Mish aktivasyon fonksiyonu tüm evrişim katmanlarında kullanılır. Eş zamanlı olarak spektral-uzamsal özellik haritalarının sayısını artırmak için 3B evrişim işlemi dört defa uygulanır ve giriş HUAG verilerinin spektral bilgilerini çıkış hacminde koruyabilir. 2B evrişim, HUAG verileri için çok önemli olan, spektral bilgide önemli bir kayıp olmaksızın, farklı spektral bantlardaki uzamsal bilgiyi güçlü bir şekilde ayırt ettiği akılda tutularak, düzleştirilmiş katmandan önce iki defa uygulanır. $3 \mathrm{~B}$ ve $2 \mathrm{~B}$ Evrişim katmanlarından sonra çıkarılan özellikler düzleştirilir ve sınıflandırma için tam bağlı katmanlara girdi olarak verilir. Bu yöntemde, 256 ve 128 nöron bulunan iki tane tam bağlantılı katman kullanılmaktadır. Aşırı öğrenmeyi önlemek için, tamamen bağlı her katmandan sonra \%0.4 dropout oranına sahip dropout katmanı uygulanmaktadır. Tam bağlantılı katmanının (FC) çıktısı, gerekli sınıflandırma sonucunu oluşturmak için basit softmax sınıflandırıcıya verilmektedir. 


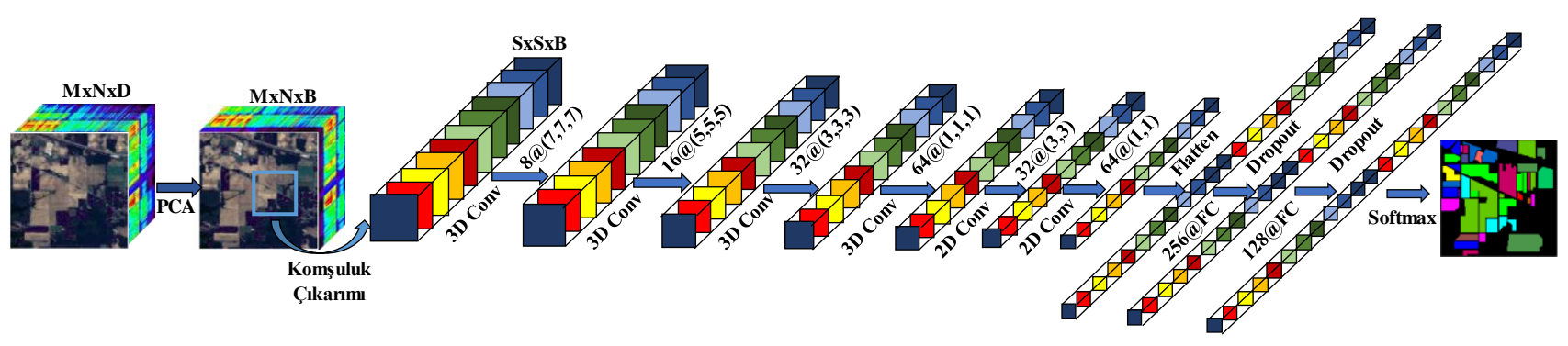

Şekil 2. Önerilen 3B-2B ESA yöntemi

\section{Verisetleri ve Uygulama Sonuçları}

\subsection{Verisetleri:}

Önerilen yöntem ile karşılaştırma için kullanılan diğer yöntemlerin performansını değerlendirmek için Indian Pines (IP) ve Pavia University (PU) hiperspektral uzaktan algilama verisetleri kullanılmaktadır. IP ve PU verisetleri http://www.ehu.eus/ccwintco/index.php/Hyperspectral_Remote_Sensing_Scenes websitesinden alınmaktadır. IP veriseti, Kuzeybatı Indiana'daki Indian Pines test alanında Havadan Görünür / Kızılötesi Görüntüleme Spektrometresi (Airborne Visible/Infrared Imaging SpectrometerAVIRIS) sensörü tarafindan elde edilmiştir. Bu verisetindeki her spektral görüntü $145 \times 145$ uzamsal boyutundadır. Sensör, 0.4-2.5 mikrometre dalga boyu aralığında toplam 224 spektral bant elde etmiştir. Bu 224 banttan, 24 bant tam su absorpsiyonu (emme) bölgesindedir ve bunlar sınıflandırma işlemi için yararlı değildir. 24 su emme bandı çıkarıldıktan sonra kalan 200 bant deneyler için kullanılmıştır. IP veri seti 16 tür arazi örtüsü ve 10.249 örnek içermektedir. PU veriseti, kuzeydoğu İtalya'daki Pavia Üniversitesi'nden Yansitıcı Optik Sistem Görüntüleme Spektrometresi (Reflective Optics System Imaging Spectrometer - ROSIS) tarafından toplanan verilerdir. Veriseti, piksel başına $1.3 \mathrm{~m}$ uzamsal çözünürlükle 340 piksel genişliğinde ve 610 piksel yüksekliğindedir. 0.43 mikrometre ile 0.86 mikrometre arasında değişen 115 dalga boyuna sahiptir. 12 gürültü bandı çıkarıldıktan sonra, kalan 103 bant deneyde kullanılmıştır. PU veriseti, 9 tür arazi örtüsü ve 42.776 örnek içermektedir. IP ve PU verisetleri ile ilgili bilgiler Tablo 1'de verilmiştir.

Tablo 1. Hiperspektral verisetlerindeki her sınıfin örnekleri ile ilgili bilgiler

\begin{tabular}{|c|c|c|c|c|c|}
\hline \multicolumn{6}{|c|}{ Indian Pines (IP) } \\
\hline No & Sinıf Adı & $\begin{array}{c}\text { Örnek } \\
\text { Sayısı }\end{array}$ & No & Sinıf Adı & $\begin{array}{c}\text { Örnek } \\
\text { Sayıs }\end{array}$ \\
\hline 1 & Alfalfa & 46 & 9 & Oats & 20 \\
\hline 2 & Corn-notill & 1428 & 10 & Soybean-notill & 972 \\
\hline 3 & Corn-mintill & 830 & 11 & Soybean-mintill & 2455 \\
\hline 4 & Corn & 237 & 12 & Soybean-clean & 593 \\
\hline 5 & Grass-pasture & 483 & 13 & Wheat & 205 \\
\hline 6 & Grass-trees & 730 & 14 & Woods & 1265 \\
\hline 7 & Grass-pasture-mowed & 28 & 15 & Buildings-grass-trees-drives & 386 \\
\hline 8 & Hay-windrowed & 478 & 16 & Stone-steel-towers & 93 \\
\hline \multicolumn{6}{|c|}{ Pavia of University (PU) } \\
\hline No & Sinıf Adı & $\begin{array}{c}\text { Örnek } \\
\text { Sayısı }\end{array}$ & No & Sinıf Adı & $\begin{array}{c}\text { Örnek } \\
\text { Sayısı }\end{array}$ \\
\hline 1 & Asphalt & 6631 & 6 & Bare soil & 5029 \\
\hline 2 & Meadows & 18649 & 7 & Bitumen & 1330 \\
\hline 3 & Gravel & 2099 & 8 & Self-blocking Bricks & 3682 \\
\hline 4 & Trees & 3064 & 9 & Shadows & 947 \\
\hline 5 & Painted metal sheets & 1345 & & & \\
\hline
\end{tabular}




\subsection{Uygulama Sonuçları:}

Tüm uygulamalar çevrim içi bir platform olan Google Colab'da gerçekleştirilmektedir. Colab, iyi bir internet hızı gerektirmektedir. Donanım hızlandırıcı olarak grafik işlem birimi (GPU) ve Tensör işlem birimi (TPU)'yu sunmaktadır. Uygulamalarımızdaki kodlar Tensör İşlem Birimi (TPU), 35 GB Rasgele Erişim Belleği (RAM) ve veri hesaplaması için 107,77 GB depolama alanı içeren python 3 masaüstü bilgisayarda çalıştırılmıştır. Tüm uygulamalarda, eğitim-test oranı \%70-30 olarak alınmıştır. Optimizasyon için 1e-06 bozunma işlevine sahip Adam optimizer ve 0.001 öğrenme oranına sahip kategorik çaprazentropi kullanılmıştır. Eğitim süreci, 256 batch size ve 100 epochs boyunca tekrar etmektedir. Softmax'ın kullanıldığı son katman hariç tüm katmanlar için bir aktivasyon işlevi olarak Mish kullanılmaktadır. İki verisetinde de, evrişim için 15x15 komşuluk çıkarımı kullanılmaktadır. Bununla birlikte, TBA tekniğinden sonra çıkarılan bantların sayısı her iki veriseti için 15 temel bileşen kullanmaktadır. Her modelin sınıflandırma performansını değerlendirmek için genel doğruluk (GD), ortalama doğruluk (OD) ve kappa istatistiği (K) kullanılmıştır. GD, test verilerindeki doğru sınıflandırılmış örnekler ile toplam test örneği sayısı arasındaki oranla hesaplanmıştır. OD her kategorinin doğruluğunun ortalama değeridir. K, temel gerçek (ground truth) haritası ile sınıflandırma haritası arasında güçlü bir anlaşma ile ilgili karşıllklı bilgi sağlayan bir istatistiksel ölçüm metriğidir. Önerilen yöntem literatürden, 2B ESA, 3B ESA, M3B-ESA, SSRN ve HybridSN gibi derin öğrenmeye dayalı HUAG sınıflandırma yöntemleri ile karşılaştırılmıştır. Karşılaştırma sonuçlarının sınıflandırma doğrulukları PU veriseti için Tablo 2'de, IP veriseti için Tablo3'te gösterilmektedir.

Tablo 2. PU veriseti için sınıflandırma sonuçları(\%)

\begin{tabular}{ccccccc}
\hline Sinıf & 2B-ESA & 3B-ESA & $\begin{array}{c}\text { M3B } \\
\text { ESA }\end{array}$ & SSRN & HybridSN & $\begin{array}{c}\text { 3B-2B } \\
\text { ESA }\end{array}$ \\
\hline $\mathbf{1}$ & 98,51 & 98,40 & 98,31 & 100 & 100 & $\mathbf{1 0 0}$ \\
$\mathbf{2}$ & 99,54 & 96,91 & 96,10 & 99,87 & 100 & $\mathbf{1 0 0}$ \\
$\mathbf{3}$ & 84,62 & 97,05 & 96,34 & 100 & 100 & $\mathbf{1 0 0}$ \\
$\mathbf{4}$ & 98,04 & 98,84 & 98,82 & 100 & 99,84 & $\mathbf{1 0 0}$ \\
$\mathbf{5}$ & 100 & 100 & 99,97 & 100 & 100 & $\mathbf{1 0 0}$ \\
$\mathbf{6}$ & 97,10 & 99,32 & 99,83 & 100 & 100 & $\mathbf{1 0 0}$ \\
$\mathbf{7}$ & 95,05 & 98,92 & 99,66 & 100 & 100 & $\mathbf{1 0 0}$ \\
$\mathbf{8}$ & 96,39 & 98,33 & 99,23 & 99,34 & 99,98 & $\mathbf{1 0 0}$ \\
$\mathbf{9}$ & 99,69 & 99,90 & 99,92 & 100 & 99,90 & $\mathbf{1 0 0}$ \\
\hline GD & $97,86 \pm 0,2$ & $96,53 \pm 0,1$ & $95,76 \pm 0,2$ & $99,90 \pm 0,0$ & $99,98 \pm 0,0$ & $\mathbf{1 0 0} \pm \mathbf{0 , 0}$ \\
OD & $96,55 \pm 0,0$ & $97,57 \pm 1,3$ & $95,08 \pm 1,2$ & $99,91 \pm 0,0$ & $99,97 \pm 0,0$ & $\mathbf{1 0 0} \pm \mathbf{0 , 0}$ \\
$\mathbf{K}$ & $97,16 \pm 0,5$ & $95,51 \pm 0,2$ & $94,50 \pm 0,2$ & $99,87 \pm 0,0$ & $99,98 \pm 0,0$ & $\mathbf{1 0 0} \pm \mathbf{0 , 0}$ \\
\hline
\end{tabular}


Tablo 3. IP veriseti için sınıflandırma sonuçları(\%)

\begin{tabular}{ccccccc}
\hline Sinıf & 2B-ESA & 3B-ESA & $\begin{array}{c}\text { M3B } \\
\text { ESA }\end{array}$ & SSRN & HybridSN & 3B-2B ESA \\
\hline $\mathbf{1}$ & 75,00 & 79,23 & 97,03 & 97,82 & 99,38 & $\mathbf{1 0 0}$ \\
$\mathbf{2}$ & 81,40 & 88,60 & 97,90 & 99,17 & 99,58 & $\mathbf{1 0 0}$ \\
$\mathbf{3}$ & 87,60 & 85,81 & 92,41 & 99,53 & $\mathbf{9 9 , 6 6}$ & 99,6 \\
$\mathbf{4}$ & 62,04 & 90,53 & 93,25 & 97,79 & 99,88 & $\mathbf{1 0 0}$ \\
$\mathbf{5}$ & 92,30 & 96,11 & 95,00 & 99,24 & 99,53 & $\mathbf{1 0 0}$ \\
$\mathbf{6}$ & 99,21 & 98,43 & 99,74 & 99,51 & 99,96 & $\mathbf{1 0 0}$ \\
$\mathbf{7}$ & 75,00 & 92,36 & 100 & 98,70 & 99,00 & $\mathbf{1 0 0}$ \\
$\mathbf{8}$ & 100 & 98,51 & 99,99 & 99,85 & 100 & $\mathbf{1 0 0}$ \\
$\mathbf{9}$ & 64,28 & 88,90 & 96,61 & 98,50 & 100 & $\mathbf{1 0 0}$ \\
$\mathbf{1 0}$ & 82,79 & 87,72 & 96,32 & 98,74 & 99,56 & 98,63 \\
$\mathbf{1 1}$ & 91,27 & 91,42 & 97,13 & 99,30 & 99,84 & $\mathbf{9 9 , 8 6}$ \\
$\mathbf{1 2}$ & 82,89 & 90,04 & 97,16 & 98,43 & 99,52 & $\mathbf{1 0 0}$ \\
$\mathbf{1 3}$ & 99,30 & 99,00 & 99,60 & 100 & 99,86 & $\mathbf{1 0 0}$ \\
$\mathbf{1 4}$ & 98,87 & 97,95 & 98,42 & 99,31 & 100 & $\mathbf{1 0 0}$ \\
$\mathbf{1 5}$ & 86,29 & 82,57 & 83,31 & 99,20 & 99,85 & $\mathbf{1 0 0}$ \\
$\mathbf{1 6}$ & $\mathbf{1 0 0}$ & 98,51 & $\mathbf{1 0 0}$ & 97,82 & 98,46 & $\mathbf{1 0 0}$ \\
\hline GD & $89,48 \pm 0,2$ & $91,10 \pm 0,4$ & $95,32 \pm 0,1$ & $99,19 \pm 0,3$ & $99,75 \pm 0,1$ & $\mathbf{9 9 , 8 0} \pm \mathbf{0 , 0}$ \\
OD & $86,14 \pm 0,8$ & $91,58 \pm 0,2$ & $96,41 \pm 0,7$ & $98,93 \pm 0,6$ & $99,63 \pm 0,2$ & $\mathbf{9 9 , 8 8} \pm \mathbf{0 , 0}$ \\
$\mathbf{K}$ & $87,96 \pm 0,5$ & $89,98 \pm 0,5$ & $94,70 \pm 0,2$ & $99,07 \pm 0,3$ & $99,71 \pm 0,1$ & $\mathbf{9 9 , 7 8} \pm \mathbf{0 , 0}$ \\
\hline
\end{tabular}

Tablo 2 incelendiğinde PU verisetinin tüm sınıflarında önerilen yöntemin \%100 ile en iyi sınıflandırma sonuçlarını verdiği görülmektedir. Tablo 3 'te ise IP verisetinin 3 ve 10 nolu sınıflarının haricinde diğer tüm sınıflarda en iyi sınıflandırma doğruluğunun elde edildiği görülmektedir. Ayrıca önerilen yöntem GD, OD ve K değerlendirme metrikleri göz önüne alındığında her iki verisetinde de en iyi sınıflandırma performansına sahiptir. Önerilen yönteme en yakın sonuçlar HybridSN yönteminde elde edilmiştir.

\section{Sonuç}

$\mathrm{Bu}$ çalışma kapsamında, HUAG sınıflandırma için 3B-2B ESA'dan oluşan hibrid bir yöntem önerilmiştir. 3B ESA ile uzamsal-spektral özelliklerin eş zamanlı olarak çıkarılması ve 3B ESA'dan sonra kullanılan 2B ESA ile de hesaplama karmaşıklığını azaltmak ve daha fazla uzamsal özellik öğrenmek amaçlanmaktadır. Önerilen yöntemde, yeni bir aktivasyon fonksiyonu olan Mish kullanılmaktadır. Önerilen yöntem iki farklı veriseti kullanılarak literatürden 5 farklı derin öğrenme tabanlı yöntem ile karşılaştırılmıştır. Gerçekleştirilen uygulamalar sonucunda önerilen yöntemin her iki verisetinde de en iyi sınıflandırma performansına sahip olduğu görülmektedir.

\section{Kaynaklar}

Ahmad, M., Khan, A.M., Mazzara, M., Distefano, S., Ali, M., Sarfraz, M.S., 2020. A Fast and Compact 3-D CNN for Hyperspectral Image Classification. IEEE Geosci. Remote Sens. Lett. 1-5. https://doi.org/10.1109/LGRS.2020.3043710

Chen, Y., Zhao, X., Jia, X., 2015. Spectral-Spatial Classification of Hyperspectral Data Based on Deep Belief Network. IEEE J. Sel. Top. Appl. Earth Obs. Remote Sens. 8, 2381-2392. https://doi.org/10.1109/JSTARS.2015.2388577

Firat, H., Hanbay, D., 2021. 3B ESA Tabanlı ResNet50 Kullanılarak Hiperspektral Görüntülerin Sinıflandırilmas1 Classification of Hyperspectral Images Using 3D CNN Based ResNet50. 2021 29th Signal Process. Commun. Appl. Conf. 6-9. https://doi.org/10.1109/SIU53274.2021.9477899

Ge, Z., Cao, G., Li, X., Fu, P., 2020. Hyperspectral Image Classification Method Based on 2D-3D CNN 
and Multibranch Feature Fusion. IEEE J. Sel. Top. Appl. Earth Obs. Remote Sens. 13, 5776-5788. https://doi.org/10.1109/JSTARS.2020.3024841

Ham, J.S., Chen, Y., Crawford, M.M., Ghosh, J., 2005. Investigation of the random forest framework for classification of hyperspectral data. IEEE Trans. Geosci. Remote Sens. 43, 492-501. https://doi.org/10.1109/TGRS.2004.842481

Jia, J., Wang, Y., Chen, J., Guo, R., Shu, R., Wang, J., 2020. Status and application of advanced airborne hyperspectral imaging technology: A review. Infrared Phys. Technol. 104, 103115. https://doi.org/10.1016/j.infrared.2019.103115

Kang, X., Duan, P., Li, S., 2020. Hyperspectral image visualization with edge-preserving filtering and principal component analysis. Inf. Fusion 57, 130-143. https://doi.org/10.1016/j.inffus.2019.12.003

Meng, Z., Li, L., Tang, X., Feng, Z., Jiao, L., Liang, M., 2019. Multipath residual network for spectralspatial hyperspectral image classification. Remote Sens. 11, 1-19. https://doi.org/10.3390/rs11161896

Mingyi He, Bo Li, H.C., 2017. Multi-scale 3D deep convolutional neural network for hyperspectral image classification. 2017 IEEE Int. Conf. Image Process. 3904-3908.

Misra, D., 2019. Mish: A Self Regularized Non-Monotonic Activation Function.

Mohan, A., Venkatesan, M., 2020. HybridCNN based hyperspectral image classification using $\begin{array}{lllll}\text { multiscale spatiospectral features. Infrared Phys. Technol. } 108 . & \end{array}$ https://doi.org/10.1016/j.infrared.2020.103326

Roy, S.K., Krishna, G., Dubey, S.R., Chaudhuri, B.B., 2019. HybridSN: Exploring 3D-2D CNN Feature Hierarchy for Hyperspectral Image Classification. arXiv 17, 277-281.

Üzen, H., Türkoğlu, M., Hanbay, D., 2021. Derin U-Net Ağ Mimarileri Kullanarak Yüzey Hata Tespiti Surface Defect Detection Using Deep U-Net Network Architectures. 2021 29th Signal Process. Commun. Appl. Conf. https://doi.org/10.1109/SIU53274.2021.9477790

Villa, A., Benediktsson, J.A., Chanussot, J., Jutten, C., 2011. Hyperspectral image classification with Independent component discriminant analysis. IEEE Trans. Geosci. Remote Sens. 49, 4865-4876. https://doi.org/10.1109/TGRS.2011.2153861

Wang, Y., Yu, W., Fang, Z., 2020. Multiple Kernel-based SVM classification of hyperspectral images by combining spectral, spatial, and semantic information. Remote Sens. 12. https://doi.org/10.3390/RS12010120

Xu, H., Zhang, H., He, W., Zhang, L., 2019. Superpixel-based spatial-spectral dimension reduction for hyperspectral imagery classification. Neurocomputing 360, 138-150. https://doi.org/10.1016/j.neucom.2019.06.023

Y. Chen, H. Jiang, C. Li, X. Jia, P.G., 2016. Deep Feature Extraction and Classification of Hyperspectral Images Based on Convolutional Neural Networks. IEEE Trans. Geosci. Remote Sens. 54, 62326251. https://doi.org/10.1109/TGRS. 2016.2584107

Zhang, L., Zhang, L., Kumar, V., 2016. Deep learning for Remote Sensing Data. IEEE Geosci. Remote Sens. Mag. 4, 22-40.

Zhong, Z., Li, J., Luo, Z., Chapman, M., 2018. Spectral-Spatial Residual Network for Hyperspectral Image Classification: A 3-D Deep Learning Framework. IEEE Trans. Geosci. Remote Sens. 56, 
847-858. https://doi.org/10.1109/TGRS.2017.2755542 